\title{
Using an Ecological Framework to Design Mobile Technologies for Pediatric Asthma Management
}

\author{
Hee Young Jeong, Rosa I. Arriaga \\ Georgia Institute of Technology \\ School of Interactive Computing and Health Systems Institute \\ $855^{\text {th }}$ Street NW, Atlanta, GA 30332, USA \\ hyj@gatech.edu, arriaga@cc.gatech.edu
}

\begin{abstract}
Mobile technologies, due to their ubiquitous nature, play an important role in supporting health care. However, it is not easy to design useful integrated mobile services without a systematic understanding of users, and this is especially true for children. Therefore, we propose a new theoretical perspective for generating design concepts in the early stage of the design process. Our ecological model is based on Ecological Systems Theory which approaches development in terms of the child's relationships and environmental context. It leverages the fact that mobile technologies are deeply involved with users' circumstances. We argue that the ecological model can provide a heuristic to help researchers understand users' needs in context and generate concepts logically and creatively. Here we explore pediatric asthma management as a case study for this model. Finally, five promising mobile technology concepts are provided as examples for further development of mobile technologies related to pediatric asthma management.
\end{abstract}

\section{Categories and Subject Descriptors}

H.5.2. [Information Systems]: User Interfaces - User-centered design; H.5.3. [Information System]: Group and Organization Interfaces - Theory and model

\section{General Terms}

Design, Human Factors, Theory

\section{Keywords}

Mobile technologies, Health, Environment, Design, Psychology, Pediatric asthma management

\section{INTRODUCTION}

Developing technologies for improvement of our everyday lives is deeply intertwined with the issue of health and environment. As a result, the trend has been that various disciplines such as public health, biomedical engineering, environmental engineering, health science, and medicine have focused on integrated systems related to web-based and mobile technologies $[10,6]$. In particular, since mobile technologies are ubiquitous, they have become common and essential tools to communicate with other people and interact with products in managing our everyday lives. For example, SMS or automatic voice message services are provided by many hospitals to remind patients of a doctor's appointment, and electronic personal health record systems are provided for user centered healthcare services [4]. However, while mobile technologies are getting more popular in our lives, and many researchers are developing mobile applications, there is a gap between mobile technology research and practical usage of the technologies for actual users in terms of readiness to access technologies. This can be especially true when the intended user is a child.

To address this issue, we propose an ecological theoretical framework for understanding users needs based on their environmental and social experience. We use pediatric asthma management to provide concrete application examples. Asthma is a chronic respiratory disease that causes coughing, wheezing, chest tightness and breathing difficulties. In United States, there are more than 22.2 million people with asthma and 6.5 million of them are children. In fact, asthma is the most common chronic disease of childhood and has high medical costs due to different medicines, medical devices, hospitalization, emergency room visits, education, and environment management. There are various asthma triggers from physical activities to environmental or emotional conditions [18]. A host of mobile technologies have been developed for asthma management. However, there has been little attention paid to integrating a holistic approach to understanding it. The need for such an approach is especially pressing when we consider the case of children with asthma, since their health is tied to their relationship with their parents, other caregivers, peers, and healthcare providers. Moreover, the environment both at home and beyond greatly affects their health.

In this paper, we first describe related work. We then explore the traditional process that researchers engage in when they consider new technology for pediatric asthma management. Next, we introduce the Ecological Systems Theory [3] and propose the ecological model. We suggest that this model takes into account many more factors than the traditional design paradigm and that it can lead to new insights for developing mobile technologies. We then provide examples for pediatric asthma management.

\section{RELATED WORK}

Many studies have explored the medical and psychological aspects of asthma management, and there are a number of relevant technologies from conventional applications to GPS. We found two major themes in the asthma management literature: 
asthma monitoring and information transfer; and environmental intervention.

Asthma monitoring and information transfer: In chronic disease management, continuous monitoring of healthcare status and regular checkup reminders are major technological solutions. The Peak Flow Meter (PFM) is an important tool that asthmatics use to monitor the current status of their lung health. Based on PFM results, parents can decide what the appropriate course of action is for a given day. Interestingly, researches related to PFM have focused on their cost effectiveness and reliability $[8,11]$ rather than how to make them easier for children to utilize.

On the information transfer side, there are several technological studies such as one that used SMS queries to get asthma diary entries and one that was a home automated telemanagement system $[1,7]$. Facilitating communication between children, parents, and healthcare providers is important with making sure that the asthma symptom reporting is essential for adequate management [13].

Environmental intervention: While the previous studies were centered on individuals, these set of studies were centered on environmental factors (such as air quality). They also include a ubiquitous warning system using a GPS navigation, a communitydriven monitoring system, and vest-based sensors that measure air pollutants in an integrated data mining system $[5,14,20]$.

\section{MOTIVATION}

Designing relevant technological solutions requires that researchers understand the recent literature and existing technologies. These solutions must be focused on users' needs and how users interact in the environment. The latter is an important consideration in pediatric asthma management because physical factors can trigger asthma attacks.

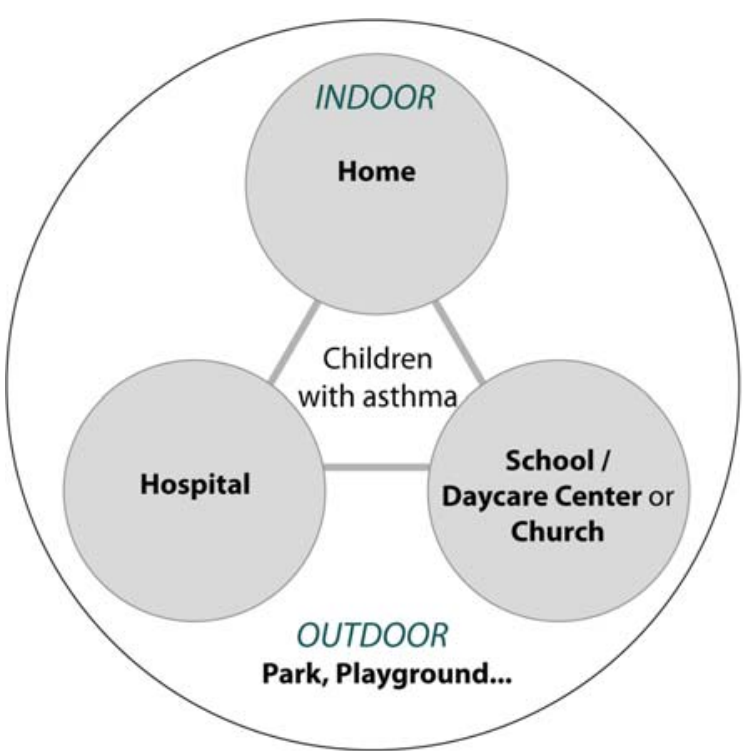

Figure 1. Initial diagram for understanding the physical environment of children with asthma

Figure 1 shows an initial conceptual diagram of the child's surroundings. However, this diagram neglects the fact that when children grow up (mentally and physically), they interact with people, products, and the environment in various ways.

Recent trends on converging paradigms encourage us to consider the interfaces in environmental and social system as a way of improving the quality of public health $[16,17]$. With this in mind, we consider Urie Bronfenbrenner's Ecological System Theory and its five interactive levels. It highlights the child's social and cultural context and her interactions with her surroundings [3]. The Microsystem embodies the child's direct experience. This has been the focus of most research in asthma management. Mesosystem shows the relation between Microsystems, for example, how a child's asthma status has influence on her school activities. Social factors indirectly related to the child are part of the Exosystem. The most far removed influence in the child's life is the Macrosystem, but it can still have profound effects on the child. This might include EPA regulations concerning emission levels. Finally, since asthma is a chronic disease, it is important to manage children's asthma symptoms over time, and this relates to the Chronosystem in Bronfenbrenner's updated ecological model (Table 1).

Table 1. Applying Bronfenbrenner's Ecological Systems Theory to pediatric asthma

\begin{tabular}{|c|l|}
\hline Levels & \multicolumn{1}{c|}{ Examples } \\
\hline Microsystem & $\begin{array}{l}\text { Child's immediate environment: Family, } \\
\text { School, Hospital (Individual entity) }\end{array}$ \\
\hline Mesosystem & $\begin{array}{l}\text { Connections between immediate } \\
\text { environment: Family to hospital, Hospital } \\
\text { to School (Relation between Microsystems) }\end{array}$ \\
\hline Exosystem & $\begin{array}{l}\text { External environment setting: Parents' } \\
\text { workplace, Activities of the local hospital } \\
\text { services (indirect relation in Microsystems } \\
\text { and Mesosystems) }\end{array}$ \\
\hline Macrosystem & $\begin{array}{l}\text { Larger cultural context: Culture, Ideology, } \\
\text { Healthcare policy, Health insurance status }\end{array}$ \\
\hline Chronosystem & $\begin{array}{l}\text { Change over time: Implications for } \\
\text { managing healthcare over time }\end{array}$ \\
\hline
\end{tabular}

We propose that Ecological Systems Theory can provide a helpful model to develop mobile technologies for children with asthma. Moreover, when we compare the territory diagram (Figure 1) with the ecological model (Table 1), we see that the territory diagram neglects the role of culture (i.e., the Macrosystem) and how the passage of time affects pediatric asthma management (i.e., the Chronosystem)

\section{ECOLOGICAL MODEL}

Here we present our ecological model for designing MobileHCI for pediatric asthma management. While there exists a number of approaches that focus interaction and interface design on the relationship between individuals and their environment (i.e. information ecology, interface ecology, cultural ecology, and product ecology $[15,12,2,9])$, none of these approaches have been applied to understanding the unique needs of pediatric users.

Figure 2 shows how an ecological framework can be used to design mobile technologies for children with asthma. The framework suggests how the five ecological layers lead to different types of technological solutions. These layers focus on 
natural relationships and frequency of interaction. We can see that the five levels refer to the individual, family, local community, society, and the environment. The order of these five layers indicates how extended relationship ranges from an individual in close and direct environment to social groups in indirect context. It is important to note that the ecological approach is socioculturally sensitive in that not all children and their environments are perceived to be the same (nor the interaction between the layers). This is important when we consider the negative effects of living in an urban or poor setting for asthma management. Thus, it reminds the designer to consider the user and the context in which the technologies may be used.

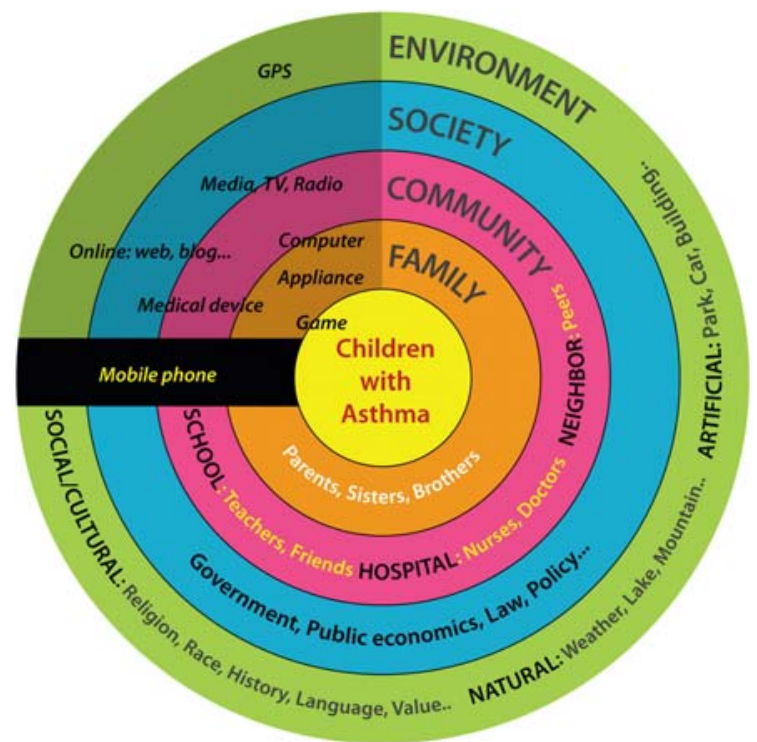

Figure 2. Ecological Model for designing mobile technologies for children with asthma

In Figure 2, the center of an ecological framework is the child (or children) with asthma. This level includes the severity of their asthma. Here children may consider themselves as asthmatics and recognize the necessity to manage their asthma status. Children may have products such as an inhaler or a PFM, but they are still under the supervision of care providers. In the second level, there is the family in the home environment. Most parents support their children's physical and emotional wellbeing and desire to take care of their children's health. Parents are also largely responsible for monitoring their child's asthma status. This may include keeping track of their child's lung capacity on a daily basis and making sure that their prescriptions are filled, that the children know where their inhalers are, and how to use them. However, parents may also be responsible for environmental (second-hand smoke) or emotional conditions (familial discord) that can increase the number of asthma attacks and severity of asthma symptoms [19]. On the other hand, the third level consists of local institutions such as hospitals and schools. At this level, children interact with teachers, friends, and healthcare providers. They have routines in different contexts, which may include meeting with healthcare providers for regular checkups or emergency visits to the hospital. They also spend some time with peers inside or outside of the home. The fourth level is similar to the Macrosystem (see Table 1). It also takes into consideration of economic trends in society. In this level, children with asthma are affected by current government's healthcare policy, public health status, and parents' economic levels. Environment is in the fifth level, and it includes natural and artificial environments including outdoor and indoor air quality. In addition, a broad range of social and cultural context is in the fifth level.

Overall, each layer includes physical, social, and cultural contexts. The main advantage of using this framework for children with asthma is the fact that each layer interplays with the others. This framework capitalizes on the dynamically distributed nature of mobile technologies and facilitates the understanding of design opportunities for pediatric asthma management.

\section{CONCEPT GENERATION}

With the understanding of users' context and viewing the related works in the framework, we generated five application concepts for asthma management through mobile technologies. These are presented in the context of the two themes identified in section 2 and prefaced by general design implications. Each concept needs to be evaluated by actual users before being implemented.

\section{General Design Implications}

Mobile technologies are uniquely suited to deliver appropriate contents to children and their parents at the various levels of the ecological approach. This can happen through automatic filtering and delivery of contents based on a crossing of context of individuals with their asthma status in the various layers. For example, automatic text messages of air quality information could be delivered based on where the child is likely to be during the week (i.e., her school's zip/postal code). This could also be synchronized to the child's schedule such that on days when she has soccer practice the information could be sent to her coach. In addition, relevant pollution index on the weekends could be based on the parent's cell phone GPS. Further, GPS and zip/postal codes can be a correlate to economic and educational status and suggest individualized information. For example, a tip of the day for controlling environmental triggers could be different for individuals living in suburban or urban areas.

\section{Asthma monitoring and information transfer:}

- Fun interactive wrist watch game (child level in the framework): In order to help children measure the maximum speed of expiration or practice breathing exercises, an interactive game with enjoyable user interfaces can be developed. It can have interesting metaphors such as pinwheels or candles to keep the child engaged. The watch is unobtrusive and useful for reminding the child to take regular measurements.

- Converged smart phone (children and community levels in the framework): All-in-one smart phone contains four major functions: peak flow meter data entry, inhaler usage log, asthma diary, and test message feedback system. People with asthma can directly log their peak flow values on the phone and log how many times they have used an inhaler. This information could be directly sent to their primary health providers and would allow for more accurate care (based on timely data). In addition, the physician could send text messages with relevant information based on data entry provided by the child. 
- Information transfer of personal health record (family and community level in the framework): In case of an emergency hospital visit, all healthcare information related to recent asthma management (i.e., type of reliever or rescue medication) could be easily and quickly retrieved from the patient's mobile phone to a central information platform via Bluetooth devices.

\section{For environmental intervention:}

- Outdoor activity support (children, community, and environment levels in the framework): A mobile phone sends an air quality warning message if children with asthma enter an area with poor air quality. In addition, the phone gives recommendations about good alternative places to move based on current user location. This recommendation service encourages users to continue outdoor activities. An alternative model for low end cell phones would allow the user to simply send a text message with their zip code. They would then get back a text message with data concerning air quality in that area.

- Indoor air quality management (family and environment levels in the ecological framework): People spend $90 \%$ of their lives in indoor environments. A mobile device or potable sensor could be set to automatically detect poor air quality and give suggestions as to how to improve it such as opening the window, thermal control, or operating ventilation system.

\section{CONCLUSION}

The purpose of this work is to introduce a new theoretical model for generating concepts in the early stage of the design process. We suggest that design and psychological perspectives can contribute to Mobile HCI research in terms of generating concepts based on both users' physical and socio-cultural environments. In our view, the ecological model is well suited to leverage the strength of mobile technology, namely, that they are naturally ubiquitous and deeply involved with users' circumstances. Our five technology concepts show that the ecological approach can help technologists design better products by encouraging them to view the impact that their technology has at various levels. While we have used pediatric asthma as our case study, we believe that the value of the ecological model extends beyond the health domain and can lead to highly improved and integrated mobile technology design for users of all ages.

\section{ACKNOWLEDGMENTS}

We would like to thank Gregory D. Abowd for the helpful advice and insight in this paper.

\section{REFERENCES}

[1] Anhøj, J. and Møldrup, C. Feasibility of Collecting Diary Data from Asthma Patients through Mobile Phones and SMS (Short Message Service): Response Rate Analysis and Focus Group Evaluation from a Pilot Study. Journal of Medical Internet Research. 2004, Vol. 6, No. 4.

[2] Bell, G. 2002. Making Sense of Museums: The Museum as Cultural Ecology. Intel Labs: Technical Report. 1-17.

[3] Bronfenbrenner, U. 1979. The Ecology of Human Development: Experiments by Nature and Design. Harvard University Press.
[4] Butz, A. and Krüger, A. User-centered development of a pervasive healthcare application. Pervasive Health Conference and Workshops, IEEE, (Nov.-Dec. 2006), 1-8.

[5] Chu, H., Huang, C., Lian, Z., and Tsai, J. A ubiquitous warning system for asthma-inducement. In Proc. of the IEEE International Conference on Sensor Networks, Ubiquitous, and Trustworthy Computing (Taichung, Taiwan, Jun. 2006), SUTC'06, IEEE, Vol. 2, 186-191.

[6] Cleland, J., Caldow J., and Ryan, D. A qualitative study of the attitudes of patients and staff to the use of mobile phone technology for recording and gathering asthma data. Journal of Telemedicine and Telecare. 2007, Vol. 13, No. 2, 85-89.

[7] Finkelstein, J. and Friedman, R.H. Telemedicine System to Support Asthma Self-Management. In Proc. of IEEE EMBS International Conference on Information Technology Applications in Biomedicine (Arlington, VA, USA, Nov. 2000), 164-167, 2000.

[8] Fonseca, J., Costa-Pereira, A., Delgado, L., Silva, L., Magalhães, M., Castel-Branco, M., and Vaz, M. Pulmonary function electronic monitoring devices. Chest. 2005, Vol. 128 , No. 3, 1258-1265.

[9] Forlizzi, J., 2007. Product ecologies: Understanding the social dynamics of products. PhD dissertation, Carnegie Mellon University.

[10] Glykasa, M. and Chytas, P. Technological innovations in asthma patient monitoring and care. Expert Systems with Applications. 2004, Vol. 27, 121-131.

[11] Kamps, A., Roorda, R., and Brand, P. Peak flow diaries in childhood asthma are unreliable. Thorax. 2001, Vol. 56, 180182.

[12] Kerne, A. 2001. A model of interface ecology. PhD dissertation. New York University.

[13] Lara, M., Duan, N., Sherbourne, C., Lewis, M., Landon, C., Halfon, N., and Brook, R. Differences between Child and Parent Reports of Symptoms among Latino Children with Asthma. Pediatrics. 1998, Vol. 102, No. 6, e68.

[14] Loh, P., Surgerman-Brozan, J., Wiggins, S., Noiles, D., and Archibald, C. From Asthma to AirBeat: Community-Driven Monitoring of Fine Particles and Carbon in Roxbury, Massachusetts. Environmental Health Perspectives. 2002, Vol. 110, Suppl. 2, 297-301.

[15] Nardi, B. and O'Day, V. 1999. Information ecologies: Using technology with heart. MIT Press.

[16] Parkes, M., Panelli, R., and Weinstein, P. Converging Paradigms for Environmental Health Theory and Practice, Environmental Health Perspectives. 2003, Vol. 111, No. 5, 669-675.

[17] Yawn, B., Fryer, G., Phillips, R., Dovey, S., Lanier, D., and Green, L. Using the ecology model to describe the impact of asthma on patterns of health care. BMC Pulmonary Medicine. 2005, 5:7.

[18] http://www.cdc.gov (07/22/2009)

[19] http://www.epa.gov/smokefree (07/22/2009)

[20] http://www.gtri.gatech.edu/casestudy (07/22/2009) 